\title{
Der Kältereiz als therapeutisches Prinzip bei Neurodermitis
}

\author{
Cold Irritation as a Therapeutical Principle in Atopic Dermatitis
}

\section{Zusammenfassung}

Die Neurodermitis (atopisches Ekzem/Dermatitis-Syndrom AEDS, atopisches oder endogenes Ekzem, atopische Dermatitis) ist auch durch eine Hyporegulation kleiner Blutgefäße mit Neigung zur Vasokonstriktion in der Haut gekennzeichnet. Eine Serie eigener Untersuchungen bestätigte entsprechende Befunde auch bei Kindern, ihre Beeinflussbarkeit durch das Nordseeklima und den therapeutischen Nutzen der Klimatherapie, auch in Hinblick auf die Normalisierung weiterer Symptome. Bei Neurodermitis ist die physiologische negative Korrelation von feuchter Abkühlungsgröße und Hautdurchblutung aufgehoben. Für Kinder sind zwei Reaktionsweisen mit einem „Normalisierungseffekt" typisch. Kaltreize wirken besonders stark und führen zu einer überschießenden reaktiven Hyperämie. Die bei Neurodermitis verminderte Hautfeuchte ändert sich auch unter Klimatherapie nicht. Symptomfreie Patienten sollten genauso intensiv behandelt werden wie solche mit Beugenekzem oder generalisierter Erkrankung. Der Dermographismus zeigt insbesondere bei ausgedehntem Hautbefall unter Klimatherapie eine Verschiebung von weiß nach rot. Eine Besserung der Hyporegulation kleiner Blutgefäße, der Neigung zur Vasokonstriktion und damit des Hautbefundes kann durch intensive Kaltreize wie Luftbäder bei böigem Wind und Seebäder erzielt werden.

\section{Abstract}

Atopic dermatitis (atopic eczema/dermatitis syndrome - AEDS, atopic or endogeneous dermatitis resp. eczema, neurodermitis) also is characterized by hyporegulation of small blood vessels with tendency to vasoconstriction in the skin. It is reported on own investigations confirming these findings in children, the influence of the North Sea climate on the regulation of skin blood flow and the therapeutical effect of the climatotherapy, also with regard to the normalization of further symptoms. In atopic dermatitis the physiological negative correlation between the humid cooling effect and the skin circulation is neutralized. In children two ways of reaction with an „effect of normalization“ are typical. Cold stimuli are outstanding effective and result in an intensive reactive hyperaemia. The diminished humidity of the skin does not change under climatotherapy. Patients without skin symptoms should be treated as intensive as those with eczema of the joints or generalized eczema. Under treatment the dermatographism shows especially in patients with widespread atopic dermatitis a shift from white to red. Improvement of hyporegulation of small blood vessels, of their tendency towards constriction and thereby of skin symptoms can be achieved by intensive cold stimuli like air baths in gusty wind or baths in the sea.

\section{Einführung}

Neuere immunologische Untersuchungen zeigen, dass Kaltreize das Immunsystem des Menschen stimulieren und dass dieses Phänomen durch Körperübungen verstärkt werden kann [1]. Dagegen unterdrücken sehr starke Kälteeinwirkungen bei Kleinsäu- gern eine Reihe von Immunreaktionen, u.a. die Vermehrung von Lymphozyten, die Bildung von natürlichen Killerzellen, zytolytische Aktivität, Komplementaktivierung und die Induktion von Hitzeschutzproteinen [2]. Von den Winterschwimmern wurde bekannt, dass deren Abwehrpotenzial gegen oxidativen Stress erhöht ist [3]. Vor diesem Hintergrund gewinnen nahezu in Ver- 
gessenheit geratene Erkenntnisse über die Verbesserung der bei Neurodermitis, neuerdings als atopic eczema/dermatitis syndrome (AEDS) bezeichnet [4], gestörten Gefäßfunktion der Haut durch Kaltreize und deren therapeutische Nutzung an aktueller Bedeutung. Nachfolgend wird über eine Reihe eigener diesbezüglicher Untersuchungen unter Nutzung des Nordseeklimas berichtet.

Im Ergebnis seiner Untersuchungen der Neurodermitis hatte Korting [5] die veränderte periphere Kreislaufsituation mit fahlem Hautkolorit, weißem Dermographismus und hypotoner Regulationsstörung herausgestellt. Das Regulationsvermögen des Gefäßsystems war meist herabgesetzt, „hyporegulativ“ und mit Vasokonstriktorentonus, nicht aber in Starre erloschen. Ähnliche Befunde waren etwa zur gleichen Zeit auch von anderen Autoren erhoben worden [6]. Buschke hatte schon im Jahre1930 geschrieben: „Von anerkanntem Nutzen sind bei chronischen Ekzemen auch die Seebäder... Während vorangegangene medikamentöse Behandlung meist nur vorübergehende Besserung hervorbringt, heilen diese Ekzeme unter der balneologischen Behandlung oft völlig ab, ohne dass Rückfälle auftreten“ [7].

Wir wollten wissen, ob

- die Blutgefäß-Regulations-Störung auch bei Kindern nachweisbar ist,

- sich diese gegebenenfalls an der Nordsee günstig beeinflussen lässt,

- dieses gegebenenfalls als Zeichen für einen guten Behandlungserfolg verwendet werden kann und

- eventuell weitere günstige Veränderungen im Gefolge der Seeklimabehandlung nachgewiesen werden können.

Die Ergebnisse der Untersuchungen sollten in therapeutische Maßnahmen einmünden.

\section{Adaptationsmangel}

Der Fluvograph der Firma Hartmann \& Braun AG Frankfurt hat einen Messfühler zur Messung der Wärmeleitzahl (Wärmetransportzahl) von lebendem Gewebe. Die Messung der Hautdurchblutung wurde mit einem Wärmeleitelement nach Golenhofen, Hensel und Hildebrandt durchgeführt. Der Wärmetransport wird durch ein konstant aufgeheiztes Goldplättchen erzeugt, das in ein festes Medium gleicher Temperatur eingelassen ist. Der Messwert ist der Wärmeleitzahl des Mediums und der Übertemperatur des geheizten Körpers proportional und entspricht dem Zeitvolumen der Durchblutung. Die Wärmeleitzahl ist die Messgröße für den Wärmetransport in der Haut, der sich stark mit dem Zeitvolumen der Durchblutung ändert.

Es wurden Korrelationen zur feuchten Abkühlungsgröße nach Robitzsch errechnet mit der Formel: $A f=\left(0,22+0,25 \mathrm{~V}^{2,3}\right)$ $(123,7-\mathrm{H})\left[\mathrm{mg} / \mathrm{cm}^{2} \times \mathrm{sec}\right] .(\mathrm{V}=$ Windgeschwindigkeit in Knoten; $\mathrm{H}=$ Äquivalenttemperatur) [6,8]. Die feuchte Abkühlungsgröße nach Robitzsch ist ein Maß für die Abkühlung eines erwärmten Körpers durch Wind, Luftfeuchtigkeit, Lufttemperatur und Sonnenstrahlung und gibt damit die bioklimatische Reizstärke an. Die Messungen erfolgten einmal wöchentlich an 16 Kindern, Jungen und Mädchen im Alter zwischen 7 und 14 Jahren. Wegen des aufwändigen Messvorganges waren täglich nur drei Messungen möglich. Anders als im Binnenland war die Hautdurchblutung an der See geringer und sank nach dem Spaziergang in der Strandzone weiter ab. Als besonders aufschlussreich erwiesen sich die Korrelationen nach zehn Messungen pro Kind. Je höher die feuchte Abkühlungsgröße war, umso niedriger war auch die Hautdurchblutung, hier die Wärmeleitzahl. Bei Anordnung nach dem Bestimmtheitsmaß ergaben sich verschiedene Diagnosegruppen: Einerseits die Neurodermitiker, bei denen sich keinerlei nachweisbare gerichtete Reaktionen auf die Strandspaziergänge fanden. Gegensätzlich verhielten sich die Kinder der Diagnosegruppe „vegetative Dysregulation“. Besonders exakte Abhängigkeit vom Einfluss einer Abkühlung kennzeichnet eine pathologische Reaktionsbereitschaft $[8,9]$.

\section{Normalisierungseffekt}

Eine Untersuchung von Neurodermitiskindern, ebenfalls Jungen und Mädchen im Alter von 7 bis 14 Jahren, erbrachte ein weiteres anregendes Ergebnis. Vor und nach einem 25-minütigen Spaziergang in der Brandungszone wurde die Wärmescheinleitzahl Lambda $(\lambda)$ bestimmt und mit der Wärmeleitzahl Lambda null $(\lambda 0)$ - für die Durchblutung des Gewebes bei gedrosselter Blutzufuhr - über fünf Wochen dargestellt. Dargestellt sind die Mittelwerte von drei gleich großen Gruppen (Abb.1).

In Abb. 1 ist eine Tendenz zur Normalisierung der Hautdurchblutung erkennbar. Bei Kindern mit hohen Ausgangswerten gehen diese im Verlauf der Klimatherapie zurück, bei denen mit niedrigen Ausgangswerten steigen sie an. Noch deutlicher wird diese Tendenz, wenn auf die Darstellung der mittleren Gruppe verzichtet wird [10]. Aus Abb. 1 ist auch ersichtlich, dass bei der Neurodermitis im Kindesalter sowohl vermehrte Vasokonstriktion als auch verstärkte Durchblutung der Haut vorkommen kann. Das typische fahle Hautkolorit ist keine angeborene pathophysiologische Eigenheit, sondern durch den Krankheitsprozess erworben. So wurden ein pastöser Hauttyp mit feuchter, etwas ödematöser Haut und ein trockener Hauttyp mit leicht schup-

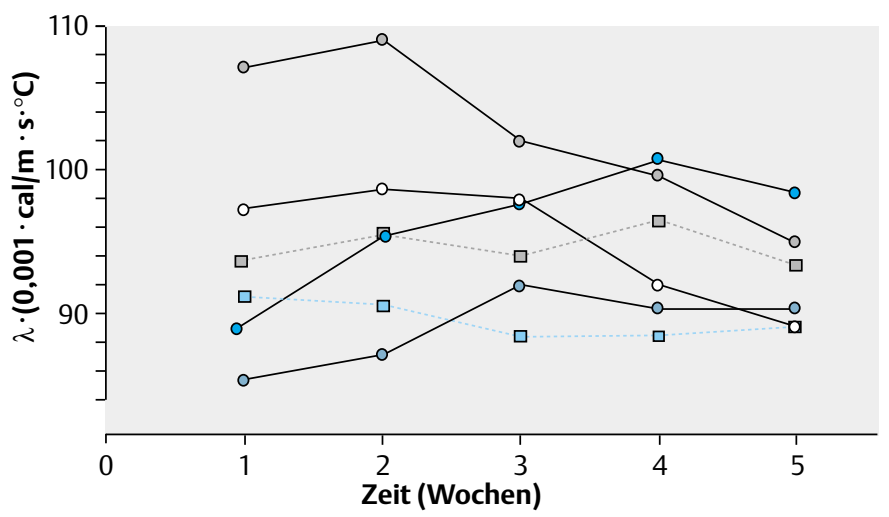

Abb. 1 Tendenz zur Normalisierung der Hautdurchblutung bei Kindern mit Neurodermitis unter der Einwirkung des Seeklimas im Verlauf von fünf Wochen. Mittelwerte der Wärmescheinleitzahl $\lambda$ und der Wärmeleitzahl $\lambda 0$ vor und nach einem Spaziergang am Strand. Abhängig von der Größe der Wärmeleitzahl vor Kurbeginn war die Aufteilung in drei gleich große Gruppen erfolgt: große, mittlere und kleine Wärmeleitzahl (Kurvenzüge von oben nach unten). 
pender, derber Haut und nur wenig Unterhautfettgewebe beschrieben [6]. Wenn die Ausgangswerte über oder unter dem jeweiligen Normalbereich liegen, sind solche „Normalisierungseffekte“ für viele Messgrößen bei Klimakuren typisch, an der See wie im Gebirge. So ließ sich eine Normalisierung der Durchblutung sogar am Nagelfalz des 5. Fingers links nachweisen [11].

\section{Vasokonstriktion}

Messungen der Hauttemperatur in verschiedenen Situationen der Klimatherapie an der See wurden mit dem Gerät „Thermorapid bzw. Lichtgalvanometer“ der Firma Netheler und Hinz durchgeführt. Das Gerät enthält einen elektrisch beheizten Thermostat mit Regler und Netztransformator für Heizung und Galvanometerbeleuchtung, Schalter zum Wählen der Betriebsart und die Anschlussbuchsen für Thermoelemente, Temperaturfühler für Messungen im Rektum, auf der Haut und der Schleimhaut der oberen Atemwege. Ein Beispiel zeigt bei Neurodermitikern im Gegensatz zu Hautgesunden bei gleichen Ausgangswerten der Temperatur einen deutlich stärkeren Abfall als Ausdruck der Neigung zur Vasokonstriktion [12] (siehe Abb. 2).

Eindrucksvoll war die Vasokonstriktion bei sehr kalten Seebädern im Monat Februar. Wegen der Kältebelastung waren zeitlich ausgedehnte Untersuchungen verständlicherweise nicht möglich. Ein Beispiel mit Gegenbeispiel gibt folgende Situation wieder: Mädchen mit Neurodermitis und mit Bronchitis hatten etwa gleiche Ausgangswerte, der Temperaturabfall bei Neurodermitikern war stärker, nach einer halben Stunde fiel die Hauttemperatur weiter ab, während bei Hautgesunden bereits ein starker Temperaturanstieg zu verzeichnen war. Trotz des verstärkten Abfalls der Hauttemperatur war bei Neurodermitikern drei Stunden später ein starker Temperaturanstieg zu verzeichnen, der deutlich höher als bei Hautgesunden war. Es liegt also keinesfalls eine Starre der Temperaturregelung in der Haut vor, sondern eine, wenn auch verzögerte, so doch erhebliche Reaktionsfähigkeit. Diese Eigenheit der Neurodermitis sollte therapeutisch genutzt werden [13].

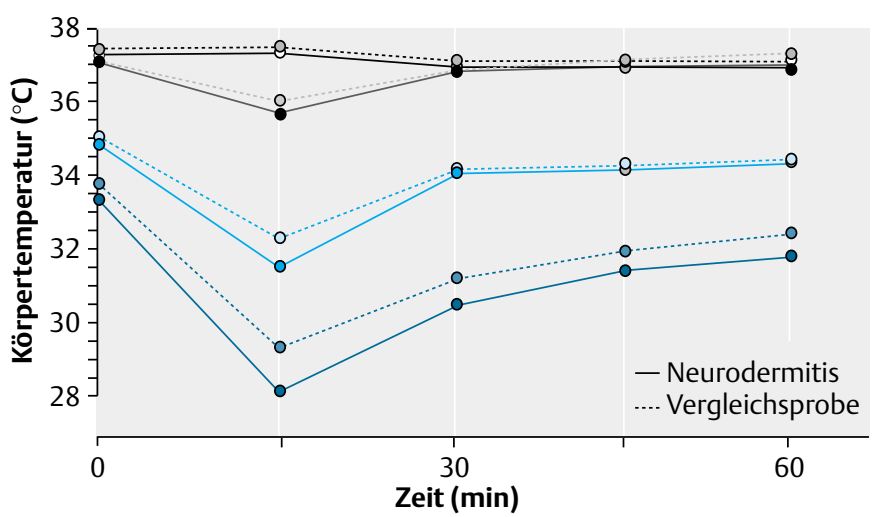

Abb. 2 Veränderungen der Temperaturen nach einem kalten Seebad bei fünf Kindern mit Neurodermitis und 15 Vergleichskindern. Messorte: rektal, sublingual, an der Stirn und integrale Hauttemperatur (Kurvenzüge von oben nach unten).

\section{Behandlungsbedarf}

Messungen verschiedener Art sollten zeigen, welche Veränderungen bei welchen pathologischen Befunden zu erwarten sind. Es handelte sich um vier Gruppen mit insgesamt 94 Kindern, Jungen und Mädchen im Alter von 5 bis 16 Jahren: I Kontrollgruppe ( $\mathrm{n}=38$ ); II latente Neurodermitis $(\mathrm{n}=14)$; III Ekzema flexuarum ( $n=19)$; IV generalisierte, stark ausgeprägte Neurodermitis $(n=23)$. Zur Messung der Hautfeuchte wurde ein Corneometer CM der Firma Schwarzhaupt verwendet, dessen Messprinzip auf Kapazitätsveränderungen durch den diffusionsabhängigen Wassergehalt im Messvolumen beruht. Die Stirnfläche des Messkopfes ist als flacher Kondensator ausgebildet, in dessen Streukapazitätsfeld (Messvolumen) die zu messende Hautoberfläche gebracht wird. Der unterschiedliche Wassergehalt der Hornschicht macht sich durch Kapazitätsänderungen bemerkbar. Die Messungen der Hautfeuchte erfolgten an 17 Hautstellen, von der Stirn bis zum Fußrücken, an den Extremitäten jeweils beidseitig. Wie zu erwarten, war die durchschnittliche Hautfeuchte bei den Kontrollkindern am höchsten und bei den Patienten mit generalisierter Neurodermitis sehr niedrig. Bemerkenswert ist jedoch, dass sich die Befunde bei Kindern mit Beugenekzem und zur Zeit symptomfreien Ekzemkindern nicht unterschieden [14]. In unserem Sinne sind auch letztere behandlungsbedürftig.

Die Alkaliresistenz und die Erythemlatenzzeit (Wiedererwärmung) der Haut änderten sich in den vier Wochen Nordseeaufenthalt nicht. Der Dermographismus der Haut war der einzige Parameter, bei dem innerhalb von nur vier Wochen eine Verschiebung der Befunde zutage trat. Bemerkenswert sind die Unterschiede zwischen Kontrollkindern und der schwersten Form, der generalisierten Neurodermitis. Bei den Kontrollkindern dominierte der rote, normale Dermographismus. Bei generalisierter Neurodermitis war anfänglich überwiegend ein pathologischer weißer Dermographismus zu verzeichnen, der sich innerhalb von vier Wochen deutlich in Richtung rote Form verschob [14]. Als Übergang von weiß zu rot kamen rasch wechselnde Befunde, auch rot mit weißem Hof oder nicht auslösbarer Dermographismus vor ( $\mathrm{n}=87$, für 4 bis 9 Wochen lückenlos) [15].

Bei hautgesunden Studenten aus Mainz ( $\mathrm{n}=12$, an 25 aufeinander folgenden Messtagen) überwog in Oberstdorf/Allgäu der weiße Dermographismus mit 59\%, ein Jahr später, bei denselben Personen und wieder im September, war es auf der Nordseeinsel Norderney jedoch der rote Dermographismus mit 83\%. Vereinzelt waren Übergangsformen zu verzeichnen [16].

\section{Konsequenzen}

Aus pathogenetischer Sicht ist Neurodermitis eine komplizierte multifaktorielle Erkrankung. Deshalb schließt ihre Therapie sehr unterschiedliche Maßnahmen ein, z.B. die Karenz potenzieller Nahrungsmittelallergene, Pseudoallergene, Kontaktsensibilisatoren und toxisch wirkender Chemikalien. Neurodermitis ist auch eine Zivilisationskrankheit, wie immer wieder betont wird. Es sollten aber nicht nur pathologische Befunde behandelt, sondern dem Krankheitsgeschehen auch der Boden entzogen werden. Hier kann eine Therapie zur Förderung der Funktionsfä- 
Tab. 1 Wirkungen kalter Brandungsbäder bei Neurodermitis

\begin{tabular}{ll}
\hline Faktor & Wirkung \\
\hline Wassertemperatur & $\begin{array}{l}\text { Vasokonstriktion } \\
\text { reaktive Vasodilatation } \\
\text { Abhärtung }\end{array}$ \\
\hline Wellen & $\begin{array}{l}\text { Anregung der Blutzirkulation } \\
\text { Straffung der Haut }\end{array}$ \\
\hline Salzgehalt & $\begin{array}{l}\text { Anregung der Durchblutung } \\
\text { Desquamation }\end{array}$ \\
\hline
\end{tabular}

Besonders bei starkem Wellengang entfalten kalte Brandungsbäder eine intensive Wirkung auf die Haut des Neurodermitikers. Wer diese Bäder - nach langsamer Eingewöhnung - vertragen kann, sollte diese vielfältigen Einflüsse wahrnehmen. Diese werden verlängert, wenn man das Salzwasser auf der Haut trocknen lässt.

higkeit der Haut ansetzen. Zunächst Vermeidung von Überwärmung, ab drittem Lebensjahr systematische Gewöhnung an Abhärtung mit kalter Luft und vom vierten Lebensjahr an mit kaltem Wasser. Abhärtung erfordert psychologisches Geschick des Therapeuten und darf dem Kranken nicht aufgezwungen werden. Neben der symptomatischen Behandlung des Ekzems ist die Normalisierung der Hautdurchblutung anzustreben. Das ist wegen häufiger Reizerscheinungen mit äußerlichen Anwendungen schwer zu erreichen. Sehr warme Wannenbäder scheiden wegen der Provokation von Juckreiz und Entzündung aus. SaunaBäder sind möglich, wenn der durch Wärme provozierte Juckreiz durch die sogleich folgende Kälte-Anwendung aufgehoben wird.

Wegen der trockenen Luft wirkt Klimatherapie im Gebirge auf Entzündungen günstig ein. Die Hyporegulation des peripheren Kreislaufsystems kann jedoch auf diese Weise nicht normalisiert werden. Klimatherapie an der Nordsee entfaltet gezielte Einwirkungen auf die Haut durch Wind, insbesondere bei Böen (Windstößen) durch starken Winddruck auf den ganzen Körper, der auf der großen Körperoberfläche aber gut vertragen wird [17,18]. Durch mäßigen Winddruck wird nach kurzer Vasokonstriktion eine anhaltende Vasodilatation erreicht, wie wir experimentell zeigen konnten [8]. Kalte Seebäder mit Wellen bedingen durch starke Druckreize eine Haut-Massage. Seebäder bei Wellengang erfordern körperliche Anstrengungen mit Leistungen von 100 Watt und weit darüber.

Durch Kälte wird Juckreiz gemildert. Für Kälteanwendungen hat sich die Gruppenbehandlung von Kindern, Jugendlichen oder
Frauen hervorragend bewährt. Gemeinsames Handeln und gegenseitiges Animieren kann zu ungeahntem persönlichen Einsatz führen, der z.B. bei sehr kalten Seebädern durch Euphorie belohnt wird [19]. Die Erfahrungen haben gezeigt, dass durch regelmäßige kalte Luft- und Seebäder auch schwere Krankheitszustände fast regelmäßig und beinahe unmerklich verschwanden (Tab. 1).

\section{Literatur}

${ }^{1}$ Brenner IK, Castellani JW, Gabaree C et al. Immune changes in humans during cold exposure: effects of prior heating an exercise. J Appl Physiol 1999; 87: 699-710

2 Shephard RJ, Shek PN. Cold exposure and immune function. Can J Physiol Pharmacol 1998; 76: 828-836

${ }^{3}$ Siems WG, Brenke R, Sommerburg 0, Grune T. Improved antioxidative protection in winter swimmers. Quart J Med 1999; 92: 193-198

${ }^{4}$ Johansson SGO, Hourihane OB, Bousquet J et al. A revised nomenclature for allergy. Allergy 2001; 56: 813-824

${ }^{5}$ Korting G. Zur Pathogenese des endogenen Ekzems. Stuttgart: Thieme, 1954

${ }^{6}$ Schüler E. Einfluss des Nordseeklimas auf die Durchblutungsgröße der Haut bei endogenen Ekzematikern. Mainz: Inaug.-Diss, 1966

${ }^{7}$ Buschke A. Balneotherapie der Haut- und Geschlechtskrankheiten. Z wiss Bäderk 1930; 4: 837-853

${ }^{8}$ Unger W. Objektivierung des Kureffektes und seines zeitlichen Ablaufs (Messung der Hautdurchblutung mit dem Fluvographen). Mainz: Inaug.-Diss, 1964

${ }^{9}$ Menger W, Unger W. Messungen der Hautdurchblutung bei Kindern während der Klimatherapie an der Nordsee. Archiv physikalische Therapie 1965; 17: $225-235$

${ }^{10}$ Menger W, Hagen M, Schüler E. Vasoneurose bei Neurodermitis constitutionalis bei Kindern und ihre Beeinflussung an der Nordsee. Z physikalische Medizin 1976; 5: 141 - 146

${ }^{11}$ Legal HP. Kapillarmikroskopische Untersuchungen bei Kindern während der Klimatherapie an der Nordsee. Mainz: Inaug.-Diss, 1966

12 Vatankhah-Barazandeh M. Haut- und Schleimhaut-Temperaturschwankungen im Nordsee-Reizklima. Mainz: Inaug.-Diss, 1972

${ }^{13}$ Menger W. Experimentelle Befunde und Erfahrungen für eine Thalassotherapie der Neurodermitis. Heilbad und Kurort 2000; 52, 3: 45-51

${ }^{14}$ Gardiewski A. Physiologische Hautfunktionsprüfungen bei Kindern mit endogenem Ekzem im Rahmen einer Klimatherapie an der Nordsee. Mainz: Inaug.-Diss, 1990

${ }^{15}$ Hagen M. Längsschnittuntersuchungen des Dermographismus bei kranken Kindern an der Nordsee. Mainz: Inaug.-Diss, 1974

${ }^{16}$ Anders R. Reaktionen der Haut unter klimatherapeutischen Bedingungen. Mainz: Inaug.-Diss, 1968

17 Menger W. Klimatherapie an Nord- und Ostsee. Jena: Fischer, 1997

${ }^{18}$ Menger W. Notwendigkeit und Chancen der Abhärtung. Schriftenreihe des Deutschen Bäderverbandes e.V. Gütersloh: Flöttmann, 1988

${ }^{19}$ Menger W. Erfolge der Abhärtung an der Nordsee. Forschungsgemeinschaft für Meeresheilkunde e.V. Heft 15. Oldenburg: Prull, 1988 